\title{
A matching method of successive approximations and its instrumentation*
}

\author{
LEIF T. SVENSSON $\div$ \\ iniversity of Stockholm and Department of Environmental \\ Hygiene, The Swedish Enviromment Protection Board \\ Box 6706, S.113 85 Stockholm. Sweden \\ and \\ KRISTOFFER SZCZYGIEL $\div \dagger$ \\ University of Stockholm \\ Box 6706, S-113 85 Stockholm. Siveden
}

The present paper reports a method of successive approximations. The technique is optimal for matching stimuli with respect to time and all Ss are forced to use the same strategy. The S's answer to the question of which is more intense, a reference or a matching stimulus, initiates a new matching stimulus according to an algorithm that bisects intervals up or down in successive trials and gives a new matching stimulus to judge. A circuitry that governs the method of successive approximations is reported. The method is exemplified by an experimental set-up for odor intensity matching

The need for the development of a convenient procedure that allows Ss to match sensory magnitudes of various kinds has long been evident in psychophysical research. Standard instrumentation and methods are available. but matching experiments in the areas of vision and audition are time consuming: in the study of olfaction, the problem is more pronounced. For example, Lindvall and Svensson (1973) found that a simple continuous adjuster used for monitoring the matching continuum was inefficient. Apparently the Ss used various strategies: Some Ss attempted to be extremely precise (it took a long time for them to find the best matching stimulus); others signaled the best match after a few trials.

In order to obtain more reliable data, we attempted to develop a method in which the strategy was the same for all Ss and the time for making a match was minimized. The best solution to the problem seemed to be a forced-choice method combined with a strategy of binary successive approximations of the matching stimulus to the reference. The principle is as follows: The S's answer to the question of which is more intense. a reference or a matching stimulus. initiates a new matching stimulus according to an algorithm that bisects intervals up or down in successive trials and provides a new matching stimulus to judge. The method is optimal

\footnotetext{
* The research was supported by a grant from the University of Stockholm and by grants to Thomas Lindvall from the Swedish Environment protection Board and to Ulf Berglund from the Swedish Council for Social Science Research. The authors wish to thank Jerome E. Singer for his valuable comments on the manuscript.

+ Now at the University of Stockholm.

$\div+$ Now at Digital Equipment Corporation.
}

with respect to time and, in addition, the same strategy is forced on all Ss.

In this report, a matching method of successive approximations and its instrumentation will be described and exemplified by an experiment for odor intensity matching. Of course, the method can be used for other sensory continua, and the circuitry described can easily be modified for other applications.

The development of the method of successive approximations can be traced to the discoverer of logarithms. John Napier (1550-1617), who also had profound insight into the usefulness of the binary number system. Napier pointed out that any number of pounds can be weighted by placing one or more of the weights-1. 2. 4. $8 \mathrm{lb}$, and so on-on the counterbalancing scale (e.g. Turnbull, 1956). A similar measurement approach was used by Baird and Vernon (1965) in a bisection experiment: The position of a point on a line segment was determined by having a person state "one" when the stimulus point appeared in the right half of the line and "zero" when in the left half. After the S's response was given, the half that appeared to contain the point was bisected, thus yielding the second digit of the sequence. The procedure continued until no more binary decisions could be made. The rationale for the present method, as well as those described above. arises from the fact that the information content of a class of stimuli can be characterized by labeling all possible members of the class with binary numbers (cf. Attneave, 1959).

\section{A MATCHING METHOD OF SUCCESSIVE APPROXIMATIONS}

The Procedure for the Subject

The $S$ is seated in front of two displays. On one of the displays a reference stimulus is presented; on the other, a matching stimulus. Signal lamps (or auditory signals) on the displays indicate the beginning and the end of a presentation. The $S$ is instructed to turn to one of the displays for a short perception of the stimulus and then to turn immediately to the other display for another perception of that stimulus. (The lights on the displays are on for a time period sufficient for the task.) Then the $\mathrm{S}$ makes a forced-choice judgment. Between presentations. the $\mathrm{S}$ must wait a certain amount of time until the lamps on the displays signal stable stimuli. The intertrial interval time is also necessary for the $S$ to adapt to neutral conditions.

The $\mathrm{S}$ is given two stimuli, a reference and a matching stimulus. The $S$ must give one of three answers in a forced-choice task: (1) The reference stimulus is the more intense. (2) The reference and matching stimulus 
Table 1

List of the Response Sequences for the Case in Which the Number of Matching Stimuli is 15

\begin{tabular}{crrr}
\hline & & Trial & \\
1 & 2 & 3 & 4 \\
\hline 8 & 12 & 14 & 15 \\
8 & 12 & 14 & 13 \\
8 & 12 & 10 & 11 \\
8 & 12 & 10 & 9 \\
8 & 4 & 6 & 7 \\
8 & 4 & 6 & 5 \\
8 & 4 & 2 & 3 \\
8 & 4 & 2 & 1 \\
\hline
\end{tabular}

are equally intense (or the $\mathrm{S}$ cannot discriminate between the intensities of the two stimuli). (3) The matching stimulus is the more intense.

At any point, if the $\mathrm{S}$ signals Answer 2, a match is obtained and the procedure terminates. If the $S$ signals Answer 1 or 3 , no match is obtained and the procedure continues. The $\mathrm{S}^{\prime} \mathrm{s}$ answer is signaled to the $\mathrm{E}$, who either accepts Answer 2 or provides a new matching stimulus according to a preset algorithm.

\section{The Procedure for the Experimenter}

For purposes of illustration, a strategy will be described for the case in which $\mathrm{E}$ wishes to determine the best matching stimulus of 15 possible:

(a) Of the 15 stimuli arranged in rank order, the $\mathrm{E}$ first presents Stimulus 8 (the middle stimulus) as a potential match.

(b) The $\mathrm{S}$ makes a decision, choosing one of the three response alternatives noted above. If he chooses the first alternative (the reference stimulus is the more intense), Stimulus 12 will be presented; if he chooses the third (the matching stimulus is more intense), Stimulus 4 will be shown. The $E$ adds or subtracts 4 on this trial, depending on the S's response.

(c) On the second trial, 2 is added to or subtracted from the result on Trial 1 . Now four outcomes are possible for Trial 3: 14, 10,6, and 2 .

(d) On Trial 3,1 is added to or subtracted from the result on Trial 2. The outcomes possible for Trial 4 are $15,13,11,9,7,5,3$, and 1 .

(e) After the judgment on the fourth trial, no more stimuli are presented. The result is given as more or less than any odd number. This means that the matching stimulus is in an interval $(\mathrm{N}-1, \mathrm{~N})$ or $(\mathrm{N}, \mathrm{N}+1)$, where $\mathrm{N}$ is the outcome on Trial 3.

If the $\mathrm{S}$ chooses Response Alternative 2 (stimuli are equal), the $E$ merely notes the result, i.e., the best matching stimulus is in the interval $(\mathrm{N}-1 / 2, \mathrm{~N}+1 / 2)$.

In the example presented, the total number of possible response sequences is 15 . A list of the possible response combinations is presented in Table 1 . If the $S$ goes through all four trials, the number of possible response sequences is eight, as listed in the table. If he goes through three trials, the number of response sequences is reduced to four (every other row in the first three columns of the table). two trials give two possible sequences, and one trial provides one possible solution (i.e., the matching stimulus number 8 ).

\section{The Algorithm}

For the general case where the number of matching stimuli is $2^{n}-1$ and $n$ is a natural number decided in advance, the strategy is the same as in the example given above. The general case is shown in Fig. 1.

In the figure, $S_{r}$ stands for the reference and $S_{m}$ for the matching stimulus. The number of binary choices is $\mathrm{n}$, and $\mathrm{i}$ is a counting variable. The successive approximation method described in Fig. 1 is divided into four parts, with two parts for the S's judgment (SJ), one for the selection of new stimuli-the control strategy (CS), and one for the result (R). The first choice is $i=n-2$. Depending on the judgment in a trial, the matching stimulus $\left(S_{m}\right)$ in the following trial will be set to $S_{m}:=S_{m} \pm 2 i$. where the plus sign is valid if the response was that the reference stimulus is perceived as more intense than the matching stimulus $\left(S_{r}>S_{m}\right)$ and

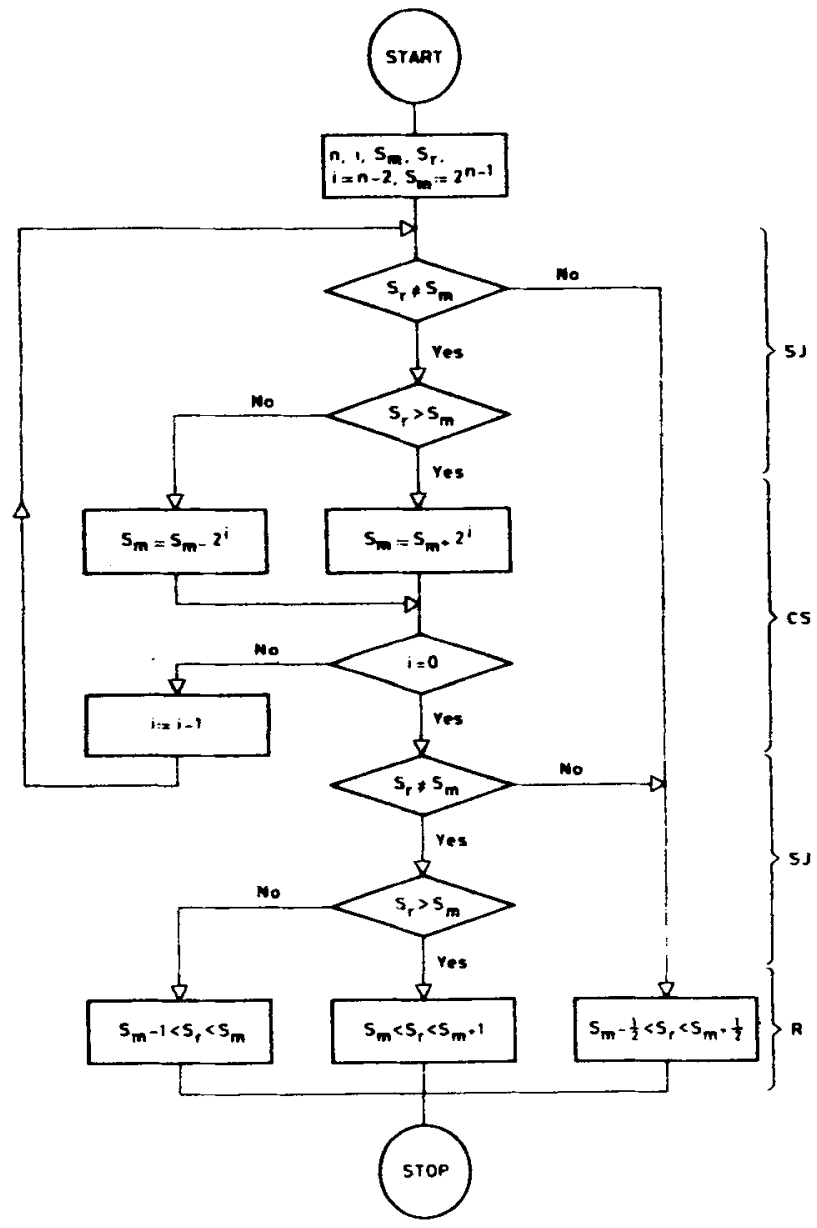

Fig. 1. Strategy of successive approximations from binary choices. [SJ = S's judgment; $C S=$ control strategy: $R=$ result $S_{m}=$ matching stimulus; $S_{x}=$ reference stimulus $i=$ counting variable; $n=$ natural number defining the total number of intervals to be $2^{n}$ and number of matching stimuli $S_{m}$ to be $2^{n}-1$. The number of effective (bounded) intervals is $2^{n}-2$.] 
in the opposite case $\left(S_{m}>S_{r}\right)$ the minus sign is valid. The algorithm counts down to $\mathrm{i}=0$. The first matching stimulus presented is $S_{m}=2 n-1$.

Referring to the example above. the number of binary choices $(\mathrm{n})$ is 4 . The number of matching stimuli is 15 $\left(=2^{n}-1\right)$ and the first matching stimulus presented is $8(=2 n-1)$. The counting variable (i) starts at 2 $(=n-2)$. In the second trial. $i=1$ : in the third trial. $\mathrm{i}=0$. In the fourth trial no more stimuli are presented (see Fig. 1). When $i=0$. depending on the answer from the $S$, the result is registered as the interval $\left(S_{m}-1\right.$. $\left.S_{m}\right)$ or $\left(S_{m} \cdot S_{m}+1\right)$. where $S_{m}$ is the outcome for the matching stimuli in Trial 3 . This means that a stimulus to match a certain reference stimulus can be found within the interval. From Fig. 1 it can also be seen that. whenever a match is obtained in a trial. the result is given as the interval $\left(S_{m}-12 . S_{m}+12\right)$. where $S_{m}$ is the outcome for the matching stimuli in the preceding trial.

\section{AN EXPERIMENTAL SET-UP FOR ODOR INTENSITY MATCHING Olfactory Laboratory}

The olfactory laboratory is constructed as a mobile unit to be used primarily in sensory evaluation of odorous air pollutants. The basic principles of the olfactometer is that it uses a well-defined dynamic flow of gas which permits stable exposure concentrations. rapid changes in concentration. and fairly natural respiratory conditions. as illustrated in Lindvall (1970). The laboratory contains a ventilated and air-conditioned test chamber in which two Teflon-coated exposure hoods have been installed. The odorant gas is mixed with purified air in fixed dilutions and added to the flow of air into the hoods by means of electromagnetically operated steel and Teflon cannulae of varying bore. The laboratory has a special waiting room for the Ss. with ventilation comparable to the actual test procedure in order to provide optimal adaptation to the experimental situation. By using suitable filter equipment (active charcoal filters and particle filters). it has been possible to carry out evaluations in an odor-free environment. Each of the odor hoods receives a continuous flow of 100 liters of purified air per minute. corresponding to a rate of approximately $0.1 \mathrm{~m} / \mathrm{sec}$.

\section{Control System for Stimulus Selection and Registration}

A control system has been developed that is programmed to perform the experimental selections and registration. It is connected to the olfactometer. as shown schematically in Fig. 2. The horizontal line dotted in the drawing divides the system into an upper and lower part: the upper part is situated in the test chamber $(\mathrm{T})$ and the lower part in the E's laboratory (L). Purified air enters the system in the laboratory, and mixed air and odorant leave the system in the test chamber. The reference and matching odorants are fed into the system in the laboratory at (1) and (m).

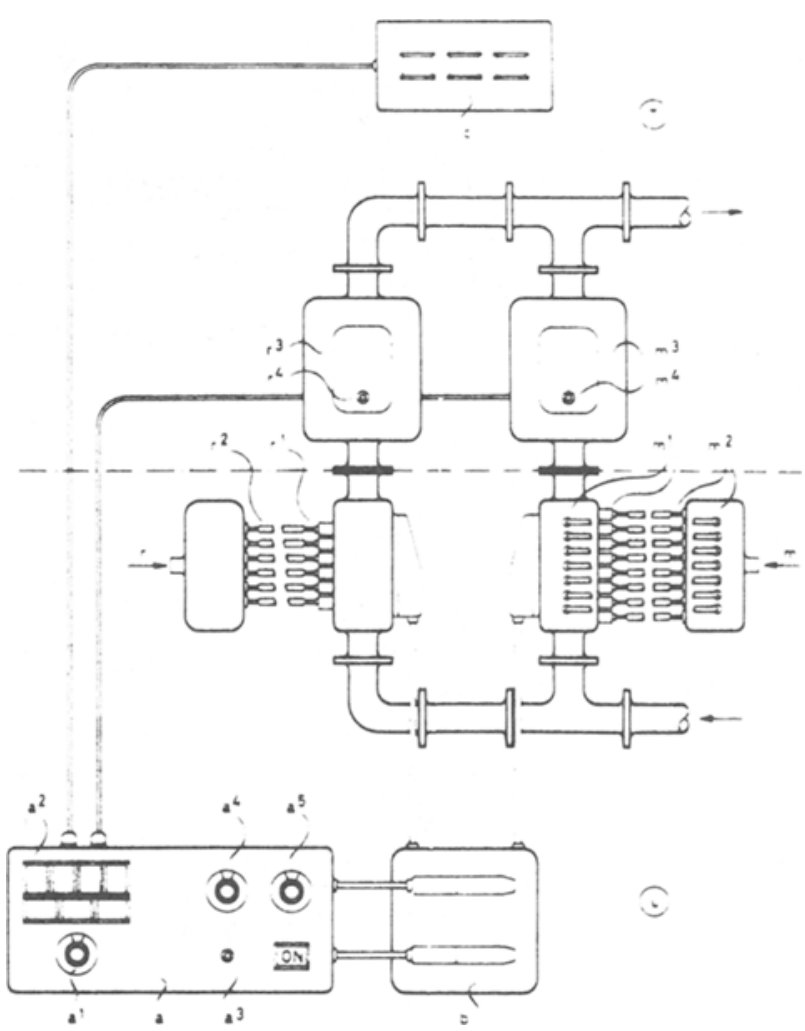

Fig. 2. Olfactometer connected to a control system of successive approximations from binary choices. $[T=$ test chamber: $L=$ laboratory: $r=$ reference system: $m=$ matching system: $a=\operatorname{logic}$ unit: $b=$ relay unit: $c=$ response panel.]

respectively. In the following. the symbol $r$ always stands for the reference s!stem and the symbol $m$ for the matching s!'stem (cf. Fig. 2).

The control system consists of three major parts: a logical unit. a relay unit. and a response panel. In Fig. 2 these are represented by the letters $a . b$, and $c$. respectively. The relay unit governs 15 electromagnetically operated valves $\left(\mathrm{m}^{1}\right)$ connected to 15 cannulae of varying bores $\left(\mathrm{m}^{2}\right)$ leading to a matching hood $\left(\mathrm{m}^{3}\right)$. With a switch $\left(\mathrm{a}^{1}\right)$ on the panel, six different reference stimuli can be selected. This switch is connected to another set of relays governing a battery of electromagnetically operated valves $\left(\mathrm{r}^{\mathrm{l}}\right)$ connected to six cannulae of differing bores $\left(\mathrm{r}^{2}\right)$ leading to a reference $\operatorname{hood}\left(r^{3}\right)$.

The result of a match to a specific reference stimulus is given by a set of seren lamps $\left(\mathrm{a}^{2}\right)$ mounted on the panel of the logical unit. the four upper lamps giving a number $(x)$ in binary digits. It the left of the lower three lamps is on. it means that the best matching stimulus is of a concentration within an interal between those given from cannulae $>-1$ and $>$. It the right lamp is on. the best matching stimulus is with an interal $(X$. $x+11$. The lamp in the middle indicates that the final

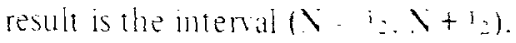




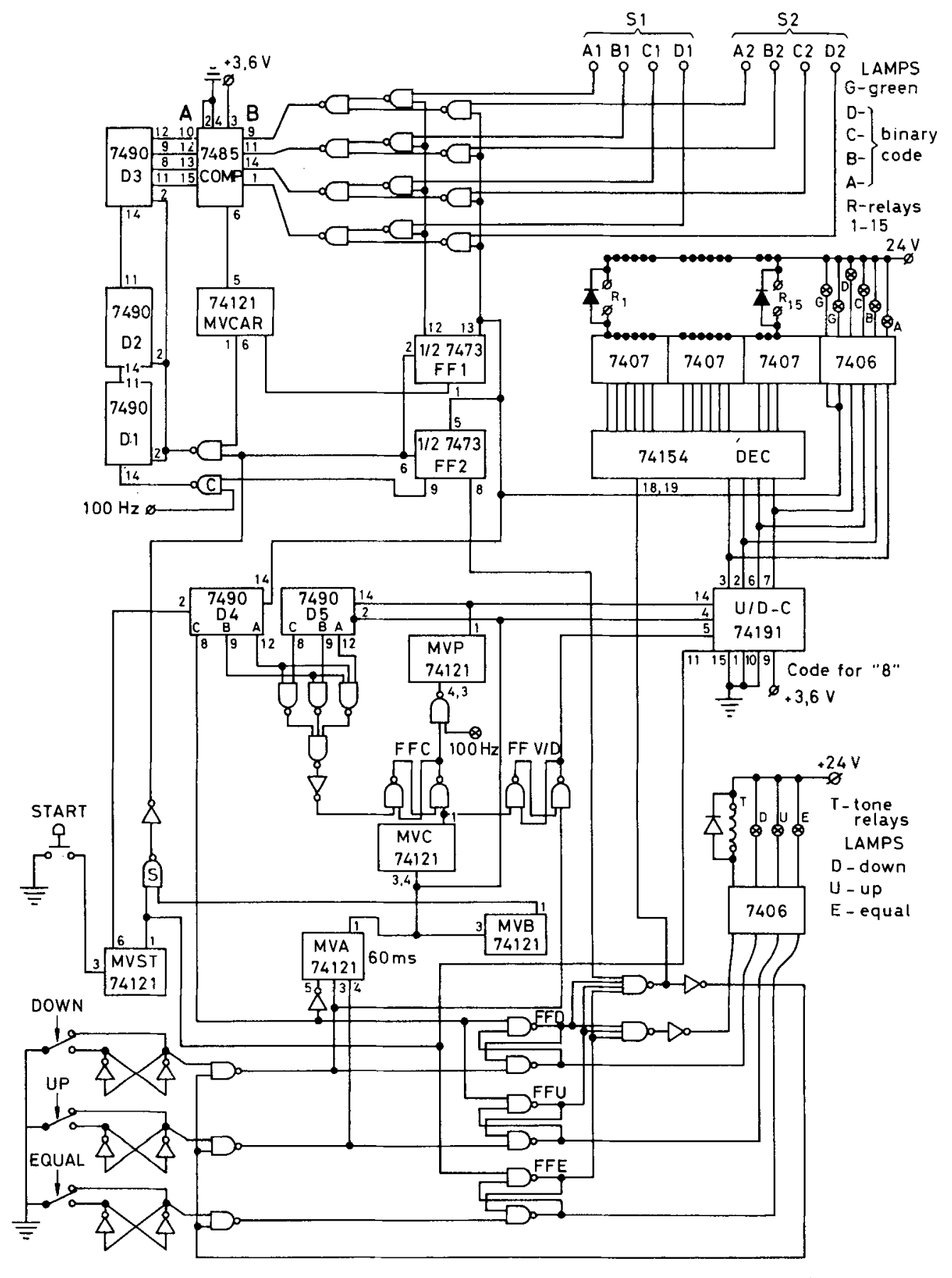

All IC's are TTL 74 series, all MV are $0,1 \mathrm{~ms}$ (excl: MVA=60ms)

Fig. 3. Circuit governing the strategy of successive approximations from binary choices.

Lamps are also mounted in the outer two of the three buttons on the response panel. With a switch $\left(\mathrm{a}^{3}\right)$ on the panel of the counting unit, the E can signal the hood from which the $S$ should start on a trial. Two timers are incorporated into the system, one for the choice of trial interval time $\left(\mathrm{a}^{4}\right)$ and one for intertrial interval time $\left(a^{5}\right)$. The last timer is connected to lamps in the odor hoods $\left(\mathrm{r}^{4}, \mathrm{~m}^{4}\right)$, which are lighted during the trial interval time. These selectors are mounted on the panel of the logical unit.

\section{Operation}

\section{Electronics of the Control System}

The circuitry and its operation is described according to Fig. 3. The logic unit operates when the E pushes the start button. The multivibrator MVST then initiates the following events:

(1) A negative pulse ( $0.1 \mathrm{msec}$ duration) that clears the flip-flops FF1 and FF2, resets the decade counters D1, D2, D3, resets the flip-flop FFE, and loads Start Number 8 into the up-and-down counter (U/D-C). The 
counter U/D.C drives. by means of the + to 16 decoder (DEC) and the relays (R). 15 electromagnetically operated valves.

(2) A positive pulse $(0.1 \mathrm{msec}$ duration) that resets the decade counter D4. The flip-flop FF1, when cleared. opens the gate system and the binary number Al-D1 goes to the "B side" of the comparator COMP. The time period $\mathrm{T} 1$ is set by Switch $\mathrm{Sl}\left(\mathrm{a}^{5}\right.$ in Fig. 2). Number $\mathrm{Al} \cdot \mathrm{D} 1$ is the desired number of seconds for the $\mathrm{Tl}$ period. Time period T2 is set by Switch S2 (Binary Number A2-D2, $a^{4}$ in Fig. 2). The flip-flop FF2 opens the clock gate $C$, and pulses of $100 \mathrm{~Hz}$ go into the decade counters (D1, D2, D3).

At every second, the output of the D3 increases by one. When the output of the D3 (the "A side" of the comparator COMP) becomes as large as Binary Number A1D1 (the "B side"). COMP changes the level" of its "A $=\mathrm{B}$ " output. which causes the multivibrator MVCAR to start. The MVCAR then gives the pulse which resets Decade Counters D1-D3 and changes the state of the flip-flop FF1. Green lamps in the odor hoods are turned on and Binary Number A2-D2 goes into the "B side" of the comparator COMP. The "A side" is now zero. Because the "B side" is not equal to zero and Clock Gate $C$ is still open (FF2"s state is unchanged), the decade counters count the new time period $\mathrm{T}_{2}$. When $\mathrm{T} 2$ time is exhausted. FF1 changes state again and (a) causes FF2 to change state (Clock Gate $C$ is closed now), (b) causes the green lamps to turn off. and $(c)$ causes D4 to change output from 0000 to 0001 . The apparatus is now in a "waiting" mode. waiting for the $\mathrm{S}$ to press one of the three buttons: up, equal, or down. Note that the response can pass because of the new state of the flip-flop FF2.)

\section{Case Equal}

The $\mathrm{S}$ presses the equal button on the response panel (c in Fig. 2). Flip-flop FFE remembers that by changing state. The lamp called equal and an acoustic signal are turned on to inform the $E$ that the experiment is terminated. and the apparatus closes all valves.

\section{Case Down}

The $\mathrm{S}$ presses the down button on the response panel (c in Fig. 2). causing the multivibrator MVA to provide a $60 \cdot \mathrm{msec}$ negative pulse. At the same time. multivibrator MVC starts and a 0.1 -msec pulse changes the state of the flip-flop FFC. The clock pulses $(100 \mathrm{~Hz})$ now go into the multivibrator MVP and then to the up-and-down counter (U/D-C) and Counter Ds:

The gate system connected to Counters D4 and D5 provides a signal when the multivibrator MVP sends a preselected number of pulses: On Trial 1 it sends four pulses, on Trial 2 two pulses. and on Trial 3 one pulse.

The pulses from the multivibrator MVP were counted down from the start value in the up-and-down counter (U/D-C). The direction of the counting depended upon the state of the flip-flop FFU/D that is set by the S. It was assumed here that the $S$ answered only if the button was depressed for more than $0.1 \mathrm{msec}$. If this assumption is changed, the circuit could be easily modified accordingly.

After $60 \mathrm{msec}$, the output of the multivibrator MVA reaches its upper iimit and the multivibrator MVB sends a $0.1-\mathrm{msec}$ negative pulse to Start Gate $S$ and begins a new trial, a new $\mathrm{T} 1$ period, etc.

\section{Case Up}

In this case. where the $S$ presses the $u p$ button on the response panel ( $c$ in Fig. 2). the response causes the up-and-down counter (U/D-C) to count up because of the state of the flip-flop FFU/D.

The logic unit holds the answer after the fourth trial by means of the flip-flops FFD and FFU. Lamps corresponding to these will be in the "on" state and an audio signal will be presented.

Note that the answer equal will be remembered by Flip-flop FFE after any one of the trials and will terminate the experiment.

\section{ADVANTAGES AND LIMITATIONS OF THE METHOD}

Although an example has been given from an olfactory laboratory, it is important to note that the method of successive approximations is independent of the sensory modality studied. The instrumentation described can. of course. be modified for other modalities. Of course, the electronic circuitry can be changed to suit the researcher's needs. We constructed a stable piece of apparatus for use in field studies that can withstand careless handling.

With $n$ binary forced-choice decisions, the strategy is optimal for finding one interval out of $2 \mathrm{n}$ possible. However, the effective range will in fact be $2^{n}-2$ intervals. If the stimuli are regarded as boundaries for the $2^{\mathrm{n}}$ intervals (the first interval is open at its lower end and the last interval is open at its upper end), then $2^{n}-2$ effective intervals for measurement remain. This means that the entire scale is limited by the first and last stimulus and must contain the reference stimulus. It can be safely fulfilled, in general, through allowing the whole scale to shift. which, in olfactory studies in particular. is accomplished by changing the pressure in the matching system of the olfactometer. Although a necessary criterion for measurement is that the stimuli on the matching scale are rank ordered. the measurement uses an interval scale only if the subjective steps between successive stimuli are equal or the number of rank ordered stimuli on a given total length of the scale is infinite.

The method of successive approximations has the same limitation as the classical method of adjustments or the method of constant stimuli: the $E$ has to choose the range of the matching continuum so that the reference stimulus can be found within the range. The great advantage of the method is that it is less time consuming 
because a minimum number of trials is used. As a consequence. the method allows $E$ to collect a large number of replications from a single $S$; also, $E$ can adjust the series of matching stimuli if the data resulting from a first series show that this is necessary. In fact, the method can be used in successive experimental steps so that a very high precision in measurement is obtained. For example. from a first series of matching stimuli $\mathrm{E}$ can obtain an interval within which the reference stimulus is located: from a second series of matching stimuli selected within this interval, the reference stimulus is further encircled, and so on.

The judgment task is plain and simple even for naive Ss, and the strategy is the same for all Ss. Cognitive, motivational, and learning factors are made less variable and will probably have smaller distortion effects on the perceptual function studied.

Thus far, two pieces have been built and adapted to olfactometers for use in the field (e.g., Berglund, Berglund. \& Lindvall, 1973). Often the Ss have been peopie living in the geographic areas, most of whom have had no previous experience with psychophysical experiments. The technique has been used also to validate the equal-sensation function and to study it more in detail. ${ }^{1,2}$ The method results in less variability and may contribute to the development of measurement models in environmental psychology.

\section{REFERENCES}

Attneave, F. Applications of information theory to psychology. New York: Holt, Rinehart \& Winston, 1959.

Baird, J. C., \& Vernon, C. W. Binary estimation. Psychonomic Science, 1965, 3, 469-470

Berglund, B., Berglund, U., \& Lindvall, T. Perceptual interaction of odors from a pulp mill. Proceedings of the Third International Clean Air Congress, Dusseldorf, October 8-12, 1973 .

Lindvall, T. On sensory evaluation of odorous air pollutant intensities. Nordisk Hygienisk Tidskrift, 1970, 2, 1-181.

Lindvall, T., \& Svensson. L. T. Equal-unpleasantness matching of malodorous substances in the community. Journal of Applied Psychology, 1973, in press.

Turnbull, H. W. The great mathematicians. In J. R. Newman (Ed.), The world of mathematics. New York: Simon \& Schuster, 1956. Swedish edition: De stora matematikerna. In T. Hall (trans.), Matematikens kulturhistoria. Stockholm: Forum, 1965

\section{NOTES}

1. Svensson, L. T., \& Lindvall, T. On the consistency of intramodal intensity matching in olfaction. Preliminary manuscript, March 1973 .

2. Svensson, L. T. An asymmetric model for the equal-sensation function in olfaction. Preliminary manuscript, A pril 1973.

(Received for publication July 16, 1973; revision received October 25,1973 .) 